\title{
Neuroendocrine Markers Insulinoma-Associated Protein 1, Chromogranin, Synaptophysin, and CD56 Show Rare Positivity in Adenocarcinoma Ex-Goblet Cell Carcinoids
}

\author{
Chen Yang ${ }^{\mathrm{a}}$, Ivan Gonzalez ${ }^{\mathrm{b}}$, Lingxin Zhang ${ }^{\mathrm{c}}$, Dengfeng Cao ${ }^{\mathrm{b}, \mathrm{d}}$
}

\begin{abstract}
Background: Adenocarcinoma ex-goblet cell carcinoid (AdexGCC) was considered a neuroendocrine adenocarcinoma, despite majority of tumor cells being negative for conventional neuroendocrine markers such as chromogranin and synaptophysin. Recently, insulinoma-associated protein 1 (INSM1) has been identified as a novel neuroendocrine marker that is more sensitive than chromogranin, synaptophysin, and CD56 in pulmonary neuroendocrine tumors.
\end{abstract}

Methods: We studied this marker in conjunction with chromogranin, synaptophysin, and CD56 in 36 appendiceal AdexGCCs (21 primaries, 15 metastatic).

Results: Primary AdexGCCs showed staining for INSM1, chromogranin, synaptophysin, and CD56 in 13/21 (62\%), 18/21 (86\%), $18 / 21(86 \%)$, and $9 / 19(47 \%)$ cases, respectively. However, the mean proportion of tumor cells stained for INSM1, chromogranin, synaptophysin, and CD56 was only $8.0 \%$ (median $1 \%$, range $0-70 \%$ ), $15.7 \%$ (median 2\%, range $0-70 \%$ ), $19.9 \%$ (median $5 \%$, range $0-90 \%$ ), and $5.6 \%$ (median $0 \%$, range $0-50 \%$ ), respectively. Metastatic AdexGCCs showed staining for INSM1, chromogranin, synaptophysin, and CD56 in 8/15 (53\%), 11/15 (73\%), 12/15 (80\%), and 3/14 (21\%) cases. The mean proportion of tumor cells stained for INSM1, chromogranin, synaptophysin, and CD56 in metastatic tumors was $1 \%$ (median $1 \%$, range $0-3 \%$ ), $12 \%$ (median $1 \%$, range $0-85 \%$ ), 17\% (median 5\%, range $0-85 \%$ ), and $2 \%$ (median $0 \%$, range $0-20 \%$ ), respectively.

Conclusions: Primary and metastatic AdexGCCs showed no difference in INSM1, chromogranin, synaptophysin, or CD56 staining. INSM1 exhibits low expression in AdexGCCs and is expressed by a

Manuscript submitted January 5, 2019, accepted January 21, 2019

aDepartment of Pathology, Yale School of Medicine, New Haven, CT 06510, USA

bDepartment of Pathology and Immunology, Washington University School of Medicine, St. Louis, MO 63110, USA

'Department of Pathology and Laboratory Medicine, Hospital for Special Surgery, New York, NY 10021, USA

${ }^{\mathrm{d}}$ Corresponding Author: Dengfeng Cao, Department of Pathology and Immunology, Washington University School of Medicine, 660 S. Euclid Ave Campus Box 8118, St. Louis, MO 63110, USA. Email: dengfengcao@wustl.edu

doi: https://doi.org/10.14740/gr1138 lower proportion of tumor cells compared to chromogranin and synaptophysin.

Keywords: Adenocarcinoma ex-goblet cell carcinoid; Neuroendocrine; Insulinoma-associated protein 1; Chromogranin; Synaptophysin

\section{Introduction}

Appendiceal goblet cell carcinoid (GCC) and adenocarcinoma ex-goblet cell carcinoid (AdexGCC) have previously been described as two entities in a spectrum of neoplasms showing both glandular/mucinous and neuroendocrine differentiation [1]. Conventional neuroendocrine markers, such as chromogranin and synaptophysin, however, are often negative or focally positive in these tumors [2,3]. Hristov et al previously demonstrated that chromogranin and synaptophysin were only expressed in $37 \%$ and $30 \%$ of AdexGCCs, respectively [2]. This is in keeping with ultrastructural studies in GCCs that showed the presence of abundant mucinous vacuoles, in comparison with the extremely difficult to locate, occasional membrane-bound, electron-dense granules [3]. In contrast, conventional carcinoids are often diffusely positive for chromogranin and synaptophysin, with abundant electron-dense granules on electron microscopy [4].

Molecular and proteomics profiling of neuroendocrine neoplasms have identified several proteins that play a pivotal role in neuroendocrine differentiation. Amongst them, one of the best characterized is insulinoma-associated protein 1 (INSM1), a zinc-finger transcription factor initially isolated from pancreatic insulinomas [5]. INSM1 is the upstream transcription factor for chromogranin [6]. As laboratory evidence began to accumulate, INSM1 has gained attention in clinical medicine as well and has been recently evaluated as a diagnostic marker of neuroendocrine differentiation in human tumor tissue samples $[7,8]$. Rosenbaum et al was the first to demonstrate INSM1 to be $92.7 \%$ sensitive and $95.8 \%$ specific in showing neuroendocrine or neuroepithelial differentiation [7]. Rooper et al further established INSM1 as a pan-neuroendocrine marker more sensitive than chromogranin, synaptophysin, and CD56 combined in pulmonary neuroendocrine neoplasms [8]. However, no studies have looked at INSM1 expression in GCCs or AdexGCCs. Studies to date have mainly focused on pure 
Table 1. Primary Adenocarcinoma Ex-Goblet Cell Carcinoid Results $(\mathrm{N}=21)$

\begin{tabular}{llllll} 
& $\mathbf{0}$ & $\mathbf{1 +}$ & $\mathbf{2 +}$ & $\mathbf{3 +}$ & $\mathbf{4 +}$ \\
\hline CG & 3 & 14 & 2 & 2 & 0 \\
SYN & 3 & 13 & 0 & 3 & 2 \\
INSM1 & 8 & 11 & 1 & 1 & 0 \\
CD56 & 10 & 8 & 1 & 0 & 0 \\
\hline
\end{tabular}

CG: chromogranin; SYN: synaptophysin. Scoring criteria: $0(<1 \%$ cells staining), $1+(1-25 \%), 2+(26-50 \%), 3+(51-75 \%)$, and $4+(76-100 \%)$.

neuroendocrine neoplasms, neglecting tumors with mixed phenotype. The purpose of this study was to investigate this novel marker in AdexGCCs, an entity thought to show mixed glandular/mucinous and neuroendocrine differentiation.

\section{Materials and Methods}

\section{Case selection}

The study was approved by the Institutional Review Board of Washington University School of Medicine. The surgical pathology archives from 1990 to 2017 of the Washington University Pathology and Immunology Department were searched for appendiceal AdexGCCs (primary and metastatic). Archived hematoxylin and eosin slides and immunohistochemistry stains were retrieved and reviewed in consensus by three pathologists (CY, LZ, and DC) to confirm the diagnosis. To ensure consistency in classification, only cases featuring both the diagnostic crypt-like architecture [1] and diffuse strong SATB2 positivity, as shown in our previous studies $[9,10]$ were included. In total, 36 appendiceal AdexGCCs (21 primaries, 15 metastases) were enrolled in our study.

\section{Immunohistochemistry}

Whole tissue paraffin blocks containing adenocarcinoma component for each case was selected. Sections were cut at $5 \mu \mathrm{m}$ thickness, deparaffinized and subjected to antigen retrieval. Immunohistochemistry was performed on all cases with monoclonal antibodies to INSM1 (Santa Cruz Biotechnology, Clone A8, 1:200 dilution), chromogranin (Ventana, clone LK2H10, prediluted), synaptophysin (Cell Marque, Clone MRQ-40, prediluted), and CD56 (Ventana, clone MRQ-42, prediluted) on a Ventana Benchmark-XT automated stainer using the Ventana ultraView DAB detection kit. Positive control (lung carcinoid) and negative control (incubation with secondary antibody only) were included for each run of immunohistochemistry stains. The tests were performed with full ethical compliance as a human study.

\section{Scoring and statistical analysis}

Only nuclear staining for INSM1, cytoplasmic staining for
Table 2. Metastatic Adenocarcinoma Ex-Goblet Cell Carcinoid Results $(\mathrm{N}=15)$

\begin{tabular}{llllll} 
& $\mathbf{0}$ & $\mathbf{1 +}$ & $\mathbf{2 +}$ & $\mathbf{3 +}$ & $\mathbf{4 +}$ \\
CG & 4 & 9 & 0 & 1 & 1 \\
SYN & 3 & 9 & 1 & 1 & 1 \\
INSM1 & 7 & 8 & 0 & 0 & 0 \\
CD56 & 11 & 3 & 0 & 0 & 0 \\
\hline
\end{tabular}

CG: chromogranin; SYN: synaptophysin. Scoring criteria: $0(<1 \%$ cells staining), $1+(1-25 \%), 2+(26-50 \%), 3+(51-75 \%)$, and $4+(76-100 \%)$.

both chromogranin and synaptophysin, and membranous staining for CD56 were considered as positive. The percentage of tumor cells stained was visually estimated and semiquantitatively scored as $0(<1 \%$ cells staining), $1+(1-25 \%)$, $2+(26-50 \%), 3+(51-75 \%)$, and $4+(76-100 \%)$. Chi-square test was used to compare the staining patterns, and Mann-Whitney $\mathrm{U}$ test to compare the mean percentage of tumor cells stained. A P-value of less than 0.05 was considered statistically significant.

\section{Results}

\section{Primary adenocarcinoma ex-goblet cell carcinoids}

Primary AdexGCCs showed staining for INSM1, chromogranin, synaptophysin, and CD56 in $13 / 21(62 \%, 1+$ in $11,2+$ in one, $3+$ in one), $18 / 21(86 \%, 1+$ in $14,2+$ in two, $3+$ in two $)$, $18 / 21(86 \%, 1+$ in $13,3+$ in three, $4+$ in two $)$, and $9 / 19(47 \%$, $1+$ in eight, $2+$ in one) cases (Table 1), respectively (INSM1 vs. chromogranin, $\mathrm{P}=0.079$; INSM1 vs. synaptophysin, $\mathrm{P}=$ 0.079; INSM1 vs. CD56, $\mathrm{P}=0.356$; chromogranin vs. synaptophysin, $\mathrm{P}=1.000$; chromogranin vs. CD56, $\mathrm{P}=0.010$; synaptophysin vs. CD56, $\mathrm{P}=0.010$ ). The mean number of tumor cells stained for INSM1, chromogranin, synaptophysin, and CD56 was $8.0 \%$ (median $1 \%$, range $0-70 \%$ ), $15.7 \%$ (median $2 \%$, range $0-70 \%$ ), $19.9 \%$ (median $5 \%$, range $0-90 \%$ ), and $5.6 \%$ (median $0 \%$, range $0-50 \%$ ), respectively (INSM1 vs. chromogranin, $\mathrm{P}=0.096$; INSM1 vs. synaptophysin, $\mathrm{P}=$ 0.021 ; INSM1 vs. CD56, $\mathrm{P}=0.433$; chromogranin vs. synaptophysin, $\mathrm{P}=0.368$; chromogranin vs. CD56, $\mathrm{P}=0.030$; synaptophysin vs. CD56, $\mathrm{P}=0.009)$. High versus low grade areas showed no difference in staining.

\section{Metastatic adenocarcinoma ex-goblet cell carcinoids}

Metastatic AdexGCCs showed staining for INSM1, chromogranin, synaptophysin, and CD56 in 8/15 (53\%, 1+ in eight), $11 / 15(73 \%, 1+$ in nine, $3+$ in one, $4+$ in one $), 12 / 15(80 \%, 1+$ in nine, $2+$ in one, $3+$ in one, $4+$ in one), and $3 / 14(21 \%, 1+$ in three) cases (Table 2), respectively (INSM1 vs. chromogranin, $\mathrm{P}=0.026$; INSM1 vs. synaptophysin, $\mathrm{P}=0.121$; INSM1 vs. $\mathrm{CD} 56, \mathrm{P}=0.077$; chromogranin vs. synaptophysin, $\mathrm{P}=0.666$; chromogranin vs. CD56, $\mathrm{P}=0.005$; synaptophysin vs. CD56, $\mathrm{P}$ 
$=0.002$ ). The mean number of tumor cells stained for INSM1, chromogranin, synaptophysin, and CD56 in metastatic tumors was $1 \%$ (median $1 \%$, range $0-3 \%$ ), $12 \%$ (median $1 \%$, range $0-85 \%$ ), $17 \%$ (median 5\%, range $0-85 \%$ ), and $2 \%$ (median $0 \%$, range $0-20 \%$ ), respectively (INSM1 vs. chromogranin, $\mathrm{P}=0.053$; INSM1 vs. synaptophysin, $\mathrm{P}=0.002$; INSM1 vs. $\mathrm{CD} 56, \mathrm{P}=0.241$; chromogranin vs. synaptophysin, $\mathrm{P}=0.166$; chromogranin vs. $\mathrm{CD} 56, \mathrm{P}=0.011$; synaptophysin vs. CD56, $\mathrm{P}=0.002)$.

\section{Comparison between primary and metastatic AdexGCCs}

Primary and metastatic AdexGCCs showed no difference in INSM1, chromogranin, synaptophysin, or CD56 staining (INSM1, $\mathrm{P}=0.30$; chromogranin, $\mathrm{P}=0.54$; synaptophysin, $\mathrm{P}$ $=0.98 ; \mathrm{CD} 56, \mathrm{P}=0.18)$. The immunohistochemistry staining results for primary and metastatic AdexGCCs are summarized in Tables 1 and 2, respectively.

\section{Discussion}

The term goblet cell carcinoid was initially coined in by Subbuswamy et al in 1974, when they described 12 morphologically distinct tumors in the vermiform appendix exhibiting histologic features different from ordinary carcinoids and colorectal adenocarcinomas [11]. Inclusion of word "carcinoid" in the nomenclature was justified by the well-differentiated appearance on histology and basal glandular position of the tumor. The term "carcinoid" was mainly adopted to emphasize the favorable prognosis. It is, however, important to take into consideration that most cases in their series were incidental findings in patients with appendicitis presenting at low clinical stage.

Two subsequent studies evaluated the neuroendocrine features of goblet cell carcinoids. Warkel et al [12] demonstrated in 1978 the lack of dysplasia in the adjacent appendiceal mucosa in these tumors. They also found concentration of tumor elements below the crypts of Lieberkuhn. Argentaffin or argyrophil granules were seen in $88 \%$ of cases. The above findings would argue that goblet cell carcinoids are different from conventional adenocarcinomas. In addition, they observed that GCCs may not behave as indolent as originally described: $15 \%$ $(6 / 39)$ of their patients developed metastatic disease. A year later, Warner et al [13] examined GCCs under electron microscopy and revealed pleomorphic neurosecretory-type granules in these tumors. They observed tumors cells of GCC which have abundant mucin to contain granules, only to a lesser extent than true neuroendocrine tumor cells. The accumulating evidence would establish goblet cell carcinoids to be partially neuroendocrine in nature.

Despite ultrastructural studies showing evidence of neurosecretory-type granules in GCCs, electron-dense granules are sparse compared to conventional carcinoids. Immunohistochemistry studies have also continuously failed to confirm extensive neuroendocrine differentiation. Conventional neuroendocrine markers, such as chromogranin and synaptophy-

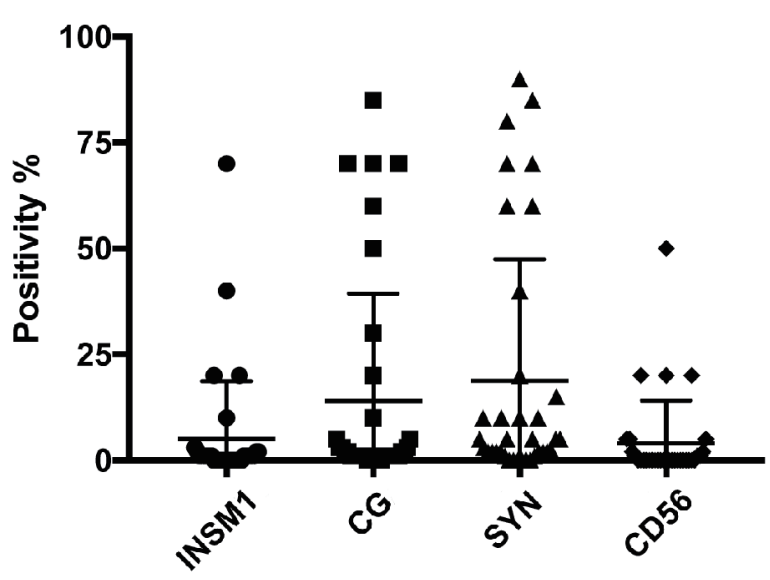

Immunohistochemical marker

Figure 1. All studied neuroendocrine markers exhibit negative to focal expression pattern in adenocarcinoma ex-goblet cell carcinoids (primary and metastatic). The majority of cases show less than $10 \%$ staining of tumor cells with any neuroendocrine marker. Synaptophysin stain shows highest percentage of positivity, followed by chromogranin, then INSM1, with CD56 showing least percentage of positive cells. CG: chromogranin; SYN: synaptophysin.

sin, have been shown to be negative or only focally positive in GCCs and AdexGCCs. Our study differs from prior approaches in three key aspects. First, we focused on AdexGCCs, which might be of more clinical relevance since systemic therapy is more often considered. Second, our study utilizes INSM1, a nuclear stain that harbor minimal background staining, which is advantageous compared to the cytoplasmic or membranous staining of conventional neuroendocrine markers, especially in mucin-rich tumors. Finally, our cases have confirmed diagnosis by both histology and immunohistochemistry $[9,10]$. In summary, our study sets to investigate the phenotype of AdexGCC using classic neuroendocrine markers chromogranin, synaptophysin, and CD56, in addition to new neuroendocrine marker INSM1.

Our results show INSM1 to be positive in 58\% (21/36) of AdexGCC cases (62\% of primary cases and $53 \%$ of metastatic cases). Furthermore, INSM1 shows positivity in only 5\% of tumor cells ( $8 \%$ in primary cases and $1 \%$ in metastatic cases). Both chromogranin and synaptophysin prove to be more sensitive when compared with INSM1. Chromogranin is positive in $81 \%(29 / 36)$ of AdexGCC cases ( $86 \%$ of primary cases and $73 \%$ of metastatic cases). It is positive in $14 \%$ of tumor cells ( $16 \%$ in primary cases and $12 \%$ in metastatic cases). Similarly, synaptophysin is positive in $83 \%(30 / 36)$ of AdexGCC cases ( $86 \%$ of primary cases and $80 \%$ of metastatic cases). A higher percentage of $19 \%$ of tumor cells $(20 \%$ in primary cases and $17 \%$ in metastatic cases) stains positive for this marker. In contrast, CD56 is the least sensitive of the four markers, being positive in only $36 \%$ (12/33) of AdexGCC cases (47\% of primary cases and $21 \%$ of metastatic cases). Rare staining is also more commonly seen with CD56, with only $4 \%$ of cells positive $(6 \%$ in primary cases and $2 \%$ in metastatic cases). The percentage of cells positive is shown in Figure 1, which shows the major- 


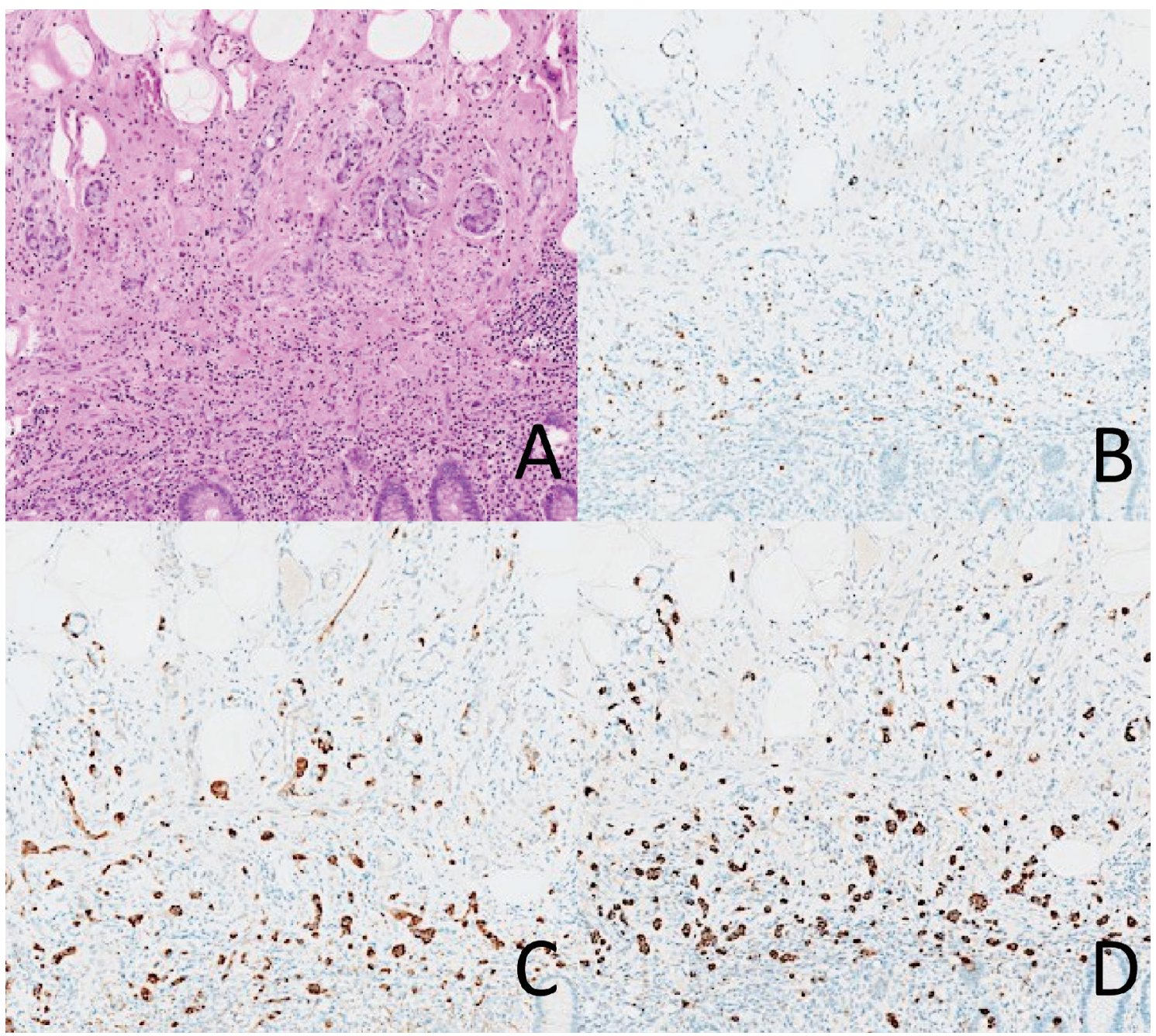

Figure 2. A case of adenocarcinoma ex-goblet cell carcinoid with positive staining for INSM1, chromogranin, and synaptophysin in the goblet cell component. There is diffuse staining of all three markers in the tumor cells. (A) Hematoxylin and eosin. (B) INSM1. (C) Chromogranin. (D) Synaptophysin.

ity of cases with less than $5 \%$ of cells positive for these neuroendocrine markers. Neuroendocrine phenotype, if present, is definitely not predominant, and would explain why neuroendocrine therapy regimens are not recommended for the treatment of AdexGCCs. As shown in our study, only five of the primary AdexGCCs show diffuse ( $>50 \%$ ) staining for any neuroendocrine marker, and only two of the metastatic AdexGCCs show diffuse $(>50 \%)$ staining for any neuroendocrine marker (Fig. 2). Most cases show either very focal positivity (Fig. 3) or are completely devoid of any neuroendocrine marker expression (Fig. 4). While there are still cases that show greater than 50\% tumor cells staining for either chromogranin or synaptophysin, both these markers are less specific in tumors with abundant mucin given their non-nuclear staining pattern. We have also observed chromogranin and synaptophysin to be positive in approximately $11 \%$ and $30 \%$ of tumor cells in gastric signet ring cell carcinomas, respectively (unpublished data).

Recent molecular studies have shown these tumors to be different genetically from conventional carcinoids and colo- rectal adenocarcinomas. Mutational landscape studies on AdexGCCs showed tumor cell mutation which were not commonly seen in colorectal adenocarcinomas such as USP9X, NOTCH1, CTNNA1, CTNNB1, TRRAP, ARID1A, ARID2, CDH1, RHPN2, MLL2, RHOA, SOX9, and KDM6A [14-16]. The mutations usually associated with colorectal adenocarcinomas, such as TP53, KRAS, and $A P C$, were only identified in rare cases of AdexGCCs [16]. Neuroendocrine tumors did not show driver mutations in one study, but this could be due to the small number of cases enrolled [14]. Johncilla et al [15] demonstrated that there is overlap in mutational landscape in GCC and AdexGCC, further evidence supporting that GCC and AdexGCC are within spectrums of the same entity, rather than two distinct histological types. Overall, the mutational landscape of GCCs and AdexGCCs are different from both conventional neuroendocrine tumors and colorectal adenocarcinomas, suggesting that they are a separate entity.

Prognostic studies also show that these tumors are distinct from neuroendocrine tumors and actually share more simi- 


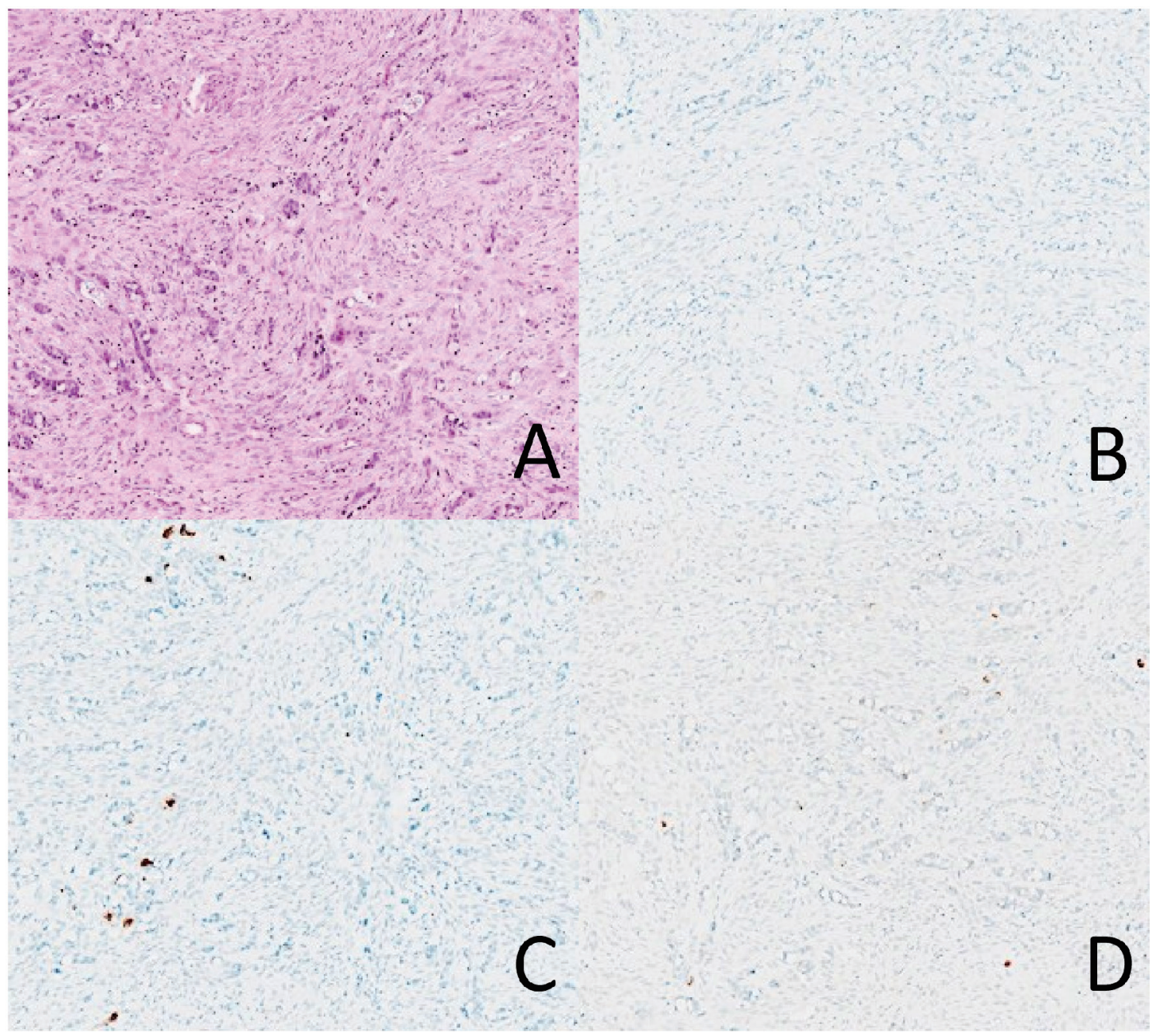

Figure 3. A case of adenocarcinoma ex-goblet cell carcinoid with weak positive staining for chromogranin and synaptophysin, but negative for INSM1. Note that chromogranin and synaptophysin are only expressed in a small proportion of tumor cells. (A) Hematoxylin and eosin. (B) INSM1. (C) Chromogranin. (D) Synaptophysin.

larities with colorectal adenocarcinomas. Most recently, Yozu et al [17] showed using their model of grading and staging AdexGCCs like other tubal gut adenocarcinomas, they can better separate patients into prognostic groups. Current surgical and oncologic approaches would favor them to be classified like an adenocarcinoma since mainstream treatment includes right hemicolectomy. The American Joint Committee on Cancer/Tumor-Node-Metastasis classification staging for AdexGCC of the appendix is similar to staging of primary adenocarcinoma of the appendix [18].

It had been shown in biological studies that cells in GCCs and AdexGCCs produce lysozyme and have a secretory component, which is more similar to intestinal crypts and not consistent with neuroendocrine cells [19]. This is in keeping with recent findings that supports the fact that GCCs and AdexGCCs have been hypothesized to origin from the crypt cells in the intestine, through differentiation through the MATH1 pathway rather than the NOTCH pathway [20,21], which can be seen in the secretory crypt cells. The same cells that are thought to dif- ferentiate through this pathway are neuroendocrine and Paneth cells. This might be one of the reasons that neuroendocrine markers such as synaptophysin and chromogranin are not proportionally, and only focally, expressed in these tumors. The hypothesis is that Paneth cells are thought to be differentiated through GFI1/SOX9 pathway, while goblet cells are differentiated through GFI1/KLF4 pathway, and finally neuroendocrine cells are differentiated with neurogenin 3 pathway. All three pathways are downstream of the MATH1 pathway. It is likely because of the close proximity in terms of the location in the tubal gut mucosa of these three cell types, and the fact that they share a common MATH1 pathway, that these three cell types can be seen intermixed in GCCs and occasionally AdexGCCs. Our study highlights the fact that AdexGCCs show either negative or limited expression of the four neuroendocrine markers studied, and in conjunction of additional evidence in molecular and prognostic studies, support separating AdexGCC from carcinoids or neuroendocrine tumor.

While our analysis is the first to comprehensively evalu- 


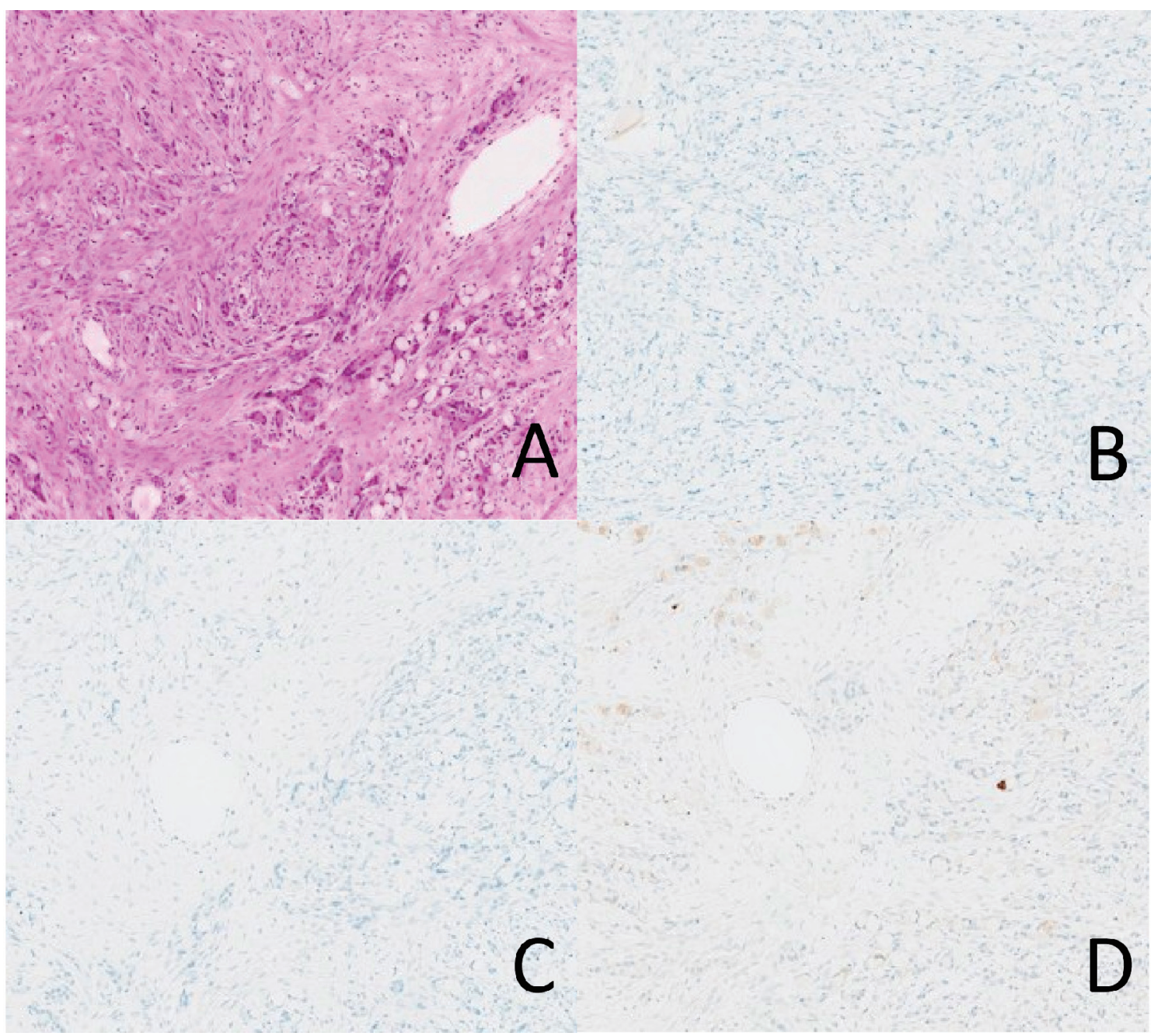

Figure 4. A case of adenocarcinoma ex-goblet cell carcinoid negative for INSM1, chromogranin, and synaptophysin. None of the tumor cells are staining with any of the markers. (A) Hematoxylin and eosin. (B) INSM1. (C) Chromogranin. (D) Synaptophysin.

ate novel neuroendocrine marker INSM1 in AdexGCCs along with three conventional neuroendocrine markers chromogranin, synaptophysin, and CD56, there are several limitations that merit discussion. First, our study was a retrospective immunohistochemistry study, and was limited by the inherent bias in studies of this type. Second, the number of cases included in our study is fairly small, due to the relatively low prevalence of these tumors. Thirdly, all the cases came from the same institution, thus a patient population selection bias could not have been avoided. Finally, follow-up information was not available for most of the cases, so the relevance of neuroendocrine marker expression and prognosis cannot be elucidated. Further prospective multi-institutional studies with more clinical intervention data would be needed to address the above issues.

\section{Conclusions}

We investigate novel neuroendocrine marker INSM1 in
AdexGCCs and found that it is often negative or shows only focal positivity in limited tumor cells. Our study highlights that INSM1, along with conventional neuroendocrine markers, such as chromogranin, synaptophysin, and CD56 shows a different staining pattern when compared with carcinoids or true neuroendocrine carcinomas. Along with emerging clinicopathologic and molecular evidence, AdexGCCs should be distinguished from a neuroendocrine tumor.

\section{Acknowledgments}

The authors would like to thank Marina Platik for her technical support with immunohistochemistry.

\section{Financial Disclosure}

The study is supported by the departmental discretionary fund from Department of Pathology and Immunology, Washington 
University School of Medicine, Saint Louis, MO.

\section{Conflict of Interest}

None of the authors have any relationships with, or financial interest in, any commercial companies pertaining to this article

\section{Informed Consent}

Informed consent was waived by the Human Research Protection Office of Washington University in St. Louis due to the retrospective nature of this study.

\section{Author Contributions}

All authors have contributed to the study. CY designed the study, collected the data, and drafted the manuscript; LZ and IG contributed to the study design and critically reviewed the manuscript; DC designed the study, oversaw the project, and critically reviewed the manuscript.

\section{References}

1. Reid MD, Basturk O, Shaib WL, Xue Y, Balci S, Choi HJ, Akkas G, et al. Adenocarcinoma ex-goblet cell carcinoid (appendiceal-type crypt cell adenocarcinoma) is a morphologically distinct entity with highly aggressive behavior and frequent association with peritoneal/intraabdominal dissemination: an analysis of 77 cases. Mod Pathol. 2016;29(10):1243-1253.

2. Hristov AC, Young RH, Vang R, Yemelyanova AV, Seidman JD, Ronnett BM. Ovarian metastases of appendiceal tumors with goblet cell carcinoidlike and signet ring cell patterns: a report of 30 cases. Am J Surg Pathol. 2007;31(10):1502-1511.

3. Kanthan R, Saxena A, Kanthan SC. Goblet cell carcinoids of the appendix: immunophenotype and ultrastructural study. Arch Pathol Lab Med. 2001;125(3):386-390.

4. Soga J, Tazawa K. Pathologic analysis of carcinoids. Histologic reevaluation of 62 cases. Cancer. 1971;28(4):990998.

5. Goto Y, De Silva MG, Toscani A, Prabhakar BS, Notkins AL, Lan MS. A novel human insulinoma-associated cDNA, IA-1, encodes a protein with "zinc-finger" DNA-binding motifs. J Biol Chem. 1992;267(21):1525215257.

6. Fujino K, Motooka Y, Hassan WA, Ali Abdalla MO, Sato Y, Kudoh S, Hasegawa K, et al. Insulinoma-associated protein 1 is a crucial regulator of neuroendocrine differentiation in lung cancer. Am J Pathol. 2015;185(12):31643177.

7. Rosenbaum JN, Guo Z, Baus RM, Werner H, Rehrauer WM, Lloyd RV. INSM1: a novel immunohistochemical and molecular marker for neuroendocrine and neuroepithelial neoplasms. Am J Clin Pathol. 2015;144(4):579-591.

8. Rooper LM, Sharma R, Li QK, Illei PB, Westra WH. INSM1 demonstrates superior performance to the individual and combined use of synaptophysin, chromogranin and CD56 for diagnosing neuroendocrine tumors of the thoracic cavity. Am J Surg Pathol. 2017;41(11):15611569.

9. Yang C, Sun L, Zhang L, Zhou L, Niu D, Cao W, Li Z, et al. SATB2 shows different profiles between appendiceal adenocarcinomas Ex goblet cell carcinoids and appendiceal/colorectal conventional adenocarcinomas: an immunohistochemical study with comparison to CDX2. Gastroenterology Res. 2018;11(3):221-230.

10. Yang C, Sun L, Zhang L, Zhou L, Zhao M, Peng Y, Niu D, et al. Diagnostic utility of SATB2 in metastatic Krukenberg tumors of the ovary: an immunohistochemical study of 70 cases with comparison to CDX2, CK7, CK20, chromogranin, and synaptophysin. Am J Surg Pathol. 2018;42(2):160-171.

11. Subbuswamy SG, Gibbs NM, Ross CF, Morson BC. Goblet cell carcinoid of the appendix. Cancer. 1974;34(2):338344.

12. Warkel RL, Cooper PH, Helwig EB. Adenocarcinoid, a mucin-producing carcinoid tumor of the appendix: a study of 39 cases. Cancer. 1978;42(6):2781-2793.

13. Warner TF, Seo IS. Goblet cell carcinoid of appendix: ultrastructural features and histogenetic aspects. Cancer. 1979;44(5):1700-1706.

14. Wen KW, Grenert JP, Joseph NM, Shafizadeh N, Huang A, Hosseini M, Kakar S. Genomic profile of appendiceal goblet cell carcinoid is distinct compared to appendiceal neuroendocrine tumor and conventional adenocarcinoma. Hum Pathol. 2018;77:166-174.

15. Johncilla M, Stachler M, Misdraji J, Lisovsky M, Yozu M, Lindeman N, Lauwers GY, et al. Mutational landscape of goblet cell carcinoids and adenocarcinoma ex goblet cell carcinoids of the appendix is distinct from typical carcinoids and colorectal adenocarcinomas. Mod Pathol. 2018;31(6):989-996.

16. Jesinghaus M, Konukiewitz B, Foersch S, Stenzinger A, Steiger K, Muckenhuber A, Gross C, et al. Appendiceal goblet cell carcinoids and adenocarcinomas ex-goblet cell carcinoid are genetically distinct from primary colorectal-type adenocarcinoma of the appendix. Mod Pathol. 2018;31(5):829-839.

17. Yozu M, Johncilla ME, Srivastava A, Ryan DP, Cusack JC, Doyle L, Setia N, et al. Histologic and outcome study supports reclassifying appendiceal goblet cell carcinoids as goblet cell adenocarcinomas, and grading and staging similarly to colonic adenocarcinomas. Am J Surg Pathol. 2018;42(7):898-910.

18. Gress DM, Edge SB, Greene FL, et al. Principles of cancer staging. AJCC Cancer Staging Manual. 8th Ed. New York: Springer-Verlag. 2017: 3-30.

19. Isaacson P. Crypt cell carcinoma of the appendix (socalled adenocarcinoid tumor). Am J Surg Pathol. $1981 ; 5(3): 213-224$.

20. van Eeden S, Offerhaus GJ, Hart AA, Boerrigter L, Ned- 
erlof PM, Porter E, van Velthuysen ML. Goblet cell carcinoid of the appendix: a specific type of carcinoma. Histopathology. 2007;51(6):763-773.
21. Crosnier C, Stamataki D, Lewis J. Organizing cell renewal in the intestine: stem cells, signals and combinatorial control. Nat Rev Genet. 2006;7(5):349-359. 